\title{
THE SPECTRUM OF SOME SPECIAL ELEMENTS IN THE FREE BANACH ALGEBRA
}

\author{
ABDULLAH H. AL-MOAJIL 1
}

ABSTRACT. Let $F$ be the free semigroup on two generators, and let $e$ denote the empty word in $F$. It is shown that if $f \in l^{\prime}(F)$ has the property that its support freely generates a subsemigroup of $F$, then the spectrum of $f$ in $l^{\prime}(F)$ is equal to $\left\{\lambda:|\lambda-f(e)| \leq \Sigma_{s \neq e}|f(s)|\right\}$.

Introduction. Let $F$ denote the free semigroup on two generators $x$ and $y$ with an identity $e$ adjoined to it ( $e$ is usually referred to as the empty word). Let $\Gamma$ be the algebra of all complex valued functions on $F$ with convolution multiplication, i. e.

$$
f g(s)=\sum_{r t=s} f(r) g(t)
$$

for all $f, g \in \Gamma$ and $s \in F$.

It is easy to see that the element of $\Gamma$ whose value is 1 at $e$ and 0 elsewhere serves as an identity for $\Gamma$, and with an easy computation one can check that an element $f \in \Gamma$ is invertible if and only if $f(e) \neq 0$.

Let $A=l^{\prime}(F)$ be the subalgebra of $\Gamma$ consisting of all $f \in \Gamma$ with the property that $\Sigma_{s \in F}|f(s)|<\infty$. Then under the usual $l^{\prime}$ norm, $A$ is a Banach algebra with identity.

Considering the generator $x$ as an element of $A$ whose value is 1 at $x$ and 0 elsewhere, it can be easily shown that $\mathrm{Sp} x=\{\lambda:|\lambda| \leq 1\}$, where Sp $x$ denotes the spectrum of $x$ in $A$. In this paper we generalize this fact to a special class of elements of $A$ that includes the generators $x$ and $y$.

Given $f \in \Gamma$ we let $S_{f}$ denote the support of $f$ in $F$, i.e. $S_{f}=\{s \in F$ : $f(s) \neq 0\}$, and we let $G_{f}$ be the subsemigroup of $F$ generated by $S_{f}$. We say $f$ is free if $G_{f}$ is freely generated by $S_{f}$. Free elements in $A$ are easily constructed, in fact every homogeneous polynomial is free [1, Chapter II], where an element $f \in \Gamma$ is a homogeneous polynomial if $S_{f}$ consists of

Received by the editors October 5, 1973 and, in revised form, February 20, 1974. AMS (MOS) subject classifications (1970). Primary 46H99; Secondary 16 A06. Key words and phrases. Free semigroup, norm, spectrum.

1The material in this paper appears in the author's Ph. D. dissertation, written under the supervision of Professor T. W. Palmer at the University of Oregon, 1973. 
words of the same degree (the degree of an element $s \in F$ is the number of occurrences of $x$ plus the number of occurrences of $y$ in the expression for $s$ ).

Lemma 1. Let $f \in \Gamma$ and suppose that $f(e) \neq 0$. Then $f$ has an inverse, $f^{-1}$, in $\Gamma$.

To prove this lemma one needs to check that there is a $g \in \Gamma$ such that $g f(e)=f g(e)=1$ and $g f(s)=f g(s)=0$ for $s \neq e$. This could be done by a straightforward computation which we omit [1, Chapter II].

Lemma 2. Let $f \in \Gamma$ with $f(e) \neq 0$. Then $S_{f^{-1}} \subset G_{f^{\circ}}$

Proof. Let $k=\min \left\{d(s): s \in S_{f} ; s \neq e\right\}$, where $d(s)$ denotes the degree of $s$.

Suppose $r \notin G_{f}$ (so in particular $r \neq e$ ) and that $d(r) \leq k_{\text {. Then }} 0=f^{-1} f(r)=$ $f^{-1}(r) f(e)$ and, hence, $f^{-1}(r)=0\left(f^{-1} f\right.$ is the identity which has value 0 except at $e$ ).

We now proceed by induction. Suppose that $f^{-1}(r)=0$ whenever $r \notin G_{f}$ and $d(r) \leq n$.

Let $d(r)=n+1$ and $r \notin G_{f}$. If $f^{-1}(r) \neq 0$, then since $f(r)=0=f^{-1} f(r)$ and $f^{-1}(r) f(e) \neq 0$, there exists $s, t \in F$ such that $s \neq e \neq t$, st $=r$ and $f^{-1}(s) f(t) \neq 0$. But then $d(s)<d(r)=n+1$. Hence, by the induction hypothesis, $s \in G_{f}$, and thus $r=s t \in G_{f}$, which is a contradiction.

Lemma 3. Let $f \in A$ and let $\lambda$ be a complex number. If $\lambda \in \mathrm{Sp} f$ then $\Sigma_{s \neq e}|f(s)| \geq \mid \lambda-f(e \mid$.

Proof. Suppose that $\Sigma_{s \neq e}|f(s)|<|\lambda-f(e)|$. Then $\lambda \neq f(e)$. Let $g=$ $[1 /(\lambda-f(e))] \cdot(\lambda-f)$. Then $g(e)=1$ and $\Sigma_{s \neq e}|g(s)|<1$. Hence

$$
\|1-g\|=|1-g(e)|+\sum_{s \neq e}|g(s)|=\sum_{s \neq e}|g(s)|<1
$$

(where $\|\cdot\|$ denotes the $l^{\prime}$ norm of $A$ ). Therefore $g$ is invertible in $A$ $[2,1.4 .5]$, and thus $\lambda-f$ is invertible :n $A$, i.e. $\lambda \not \mathrm{Sp} f$.

Lemma 4. Let $f \in A$ be free. Suppose that $S_{f}$ is finite and that $f(e)=$ 1. Then $f$ is invertible in $A$ if and only if $\Sigma_{s \neq e}|f(s)|<1$.

Proof. Since $f(e) \neq 0, f$ has an inverse, $f^{-1}$, by Lemma 1. By Lemma 2 , we have $S_{f^{-1}} \subset G_{f^{*}}$

Let $S_{f}=\left\{s_{1}, \cdots, s_{n}\right\} \cup\{e\}$. Since $f$ is free, every element in $S_{f}$ could be written uniquely in the form $t_{1} \cdots t_{m}$, where for each $i, t_{i}=s_{j}$ for some $j=1, \cdots, n$. 
We have $1=f^{-1} f(e)=f^{-1}(e) f(e)=f^{-1}(e)$.

Claim. If $s=t_{1} \cdots t_{m} \neq e, t_{i} \in S_{f}$; then $f^{-1}(s)=(-1)^{m} \Pi_{i=1}^{m} f\left(s_{i}\right)$.

We prove the claim by induction on $m$.

If $m=1$, then we have

$$
0=f^{-1} f\left(t_{1}\right)=f^{-1}\left(t_{1}\right) f(e)+f^{-1}(e) f\left(t_{1}\right)=f^{-1}\left(t_{1}\right)+f\left(t_{1}\right)
$$

(by the freeness of $f_{,} t_{1}$ cannot be written as the product of other elements of $\left.S_{f}\right)$. Hence $f^{-1}\left(t_{1}\right)=-f\left(t_{1}\right)$.

Now suppose that $f^{-1}\left(t_{1} \cdots t_{k}\right)=(-1)^{k} \Pi_{i=1}^{k} f\left(t_{i}\right)$, and let $s=t_{1} \cdots t_{k+1}$ $\neq e$. Then

$$
0=f^{-1} f(s)=f^{-1}(s) f(e)+f^{-1}\left(t_{1} \cdots t_{k}\right) f\left(t_{k+1}\right)
$$

(we used the uniqueness of the expression for $s$ as a product of elements in $S_{f}$ ). Hence

$$
\begin{aligned}
f^{-1}(s) & =-f^{-1}\left(t_{1} \cdots t_{k}\right) f\left(t_{k+1}\right) \\
& =-\left[(-1)^{k} \prod_{i=1}^{k} f\left(t_{i}\right)\right] f\left(t_{k+1}\right)=(-1)^{k+1} \prod_{i=1}^{k+1} f\left(t_{i}\right) .
\end{aligned}
$$

This proves the claim.

Now given nonnegative integers $\alpha_{1}, \cdots, \alpha_{n}$; there are

$$
\left(\alpha_{1}+\cdots+\alpha_{n}\right) ! / \alpha_{1} ! \cdots \alpha_{n} \text { ! }
$$

words in $G_{f}$ which contains $\alpha_{i}$ factors of $s_{i}$ for $i=1, \ldots, n$. All these words are distinct since $f$ is free. Hence

$$
\begin{aligned}
\left\|f^{-1}\right\| & =\sum_{a_{1}=0}^{\infty} \cdots \sum_{\alpha_{n}=0}^{\infty}\left[\frac{\left(\alpha_{1}+\cdots+\alpha_{n}\right) !}{\alpha_{1} ! \cdots \alpha_{n} !}\right]\left|f\left(s_{1}\right)^{a} \cdots f\left(s_{n}\right)^{a}{ }_{n}\right| \\
& =\sum_{p=0}^{\infty}\left(\sum_{i=1}^{n}\left|f\left(s_{i}\right)\right|\right)^{p}=\sum_{p=0}^{\infty}\left(\sum_{s \neq e}|f(s)|\right)^{p},
\end{aligned}
$$

and therefore $\left\|f^{-1}\right\|<\infty$ if and only if $\Sigma_{s \neq e}|f(s)|<1$.

Lemma 5. Let $f \in A$ be free. Suppose that $f(e)=1$. If $f$ is invertible in $A$, then $\Sigma_{s \neq e}|f(s)| \leq 1$.

Proof. Since $S_{f}$ generates a free semigroup, every subset of $S_{f}$ generates a free semigroup. Hence $f$ can be approximated by free elements with finite support. 
Let $\delta$ be a positive number less than $1 /\left\|f^{-1}\right\|$ and choose $g$ free with finite support such that $g(e)=1$ and $\|f-g\|<\delta$. Since $\delta<1 /\left\|f^{-1}\right\|, g$ is invertible in $A[2,1.4 .6]$, and thus by Lemma $4, \Sigma_{s \neq e}|g(s)|<1$. Hence

$$
\sum_{s \neq e}|f(s)|=\|f\|-1 \leq\|f-g\|+\|g\|-1=\|f-g\|+\sum_{s \neq e}|g(s)|<\delta+1 .
$$

Theorem. Let $f \in A$ be free. Then Sp $f=\left\{\lambda:|\lambda-f(e)| \leq \Sigma_{s \neq e}|f(s)|\right\}$.

Proof. By Lemma 3 we have Sp $f \subset\left\{\lambda:|\lambda-f(e)| \leq \Sigma_{s \neq e}|f(s)|\right\}$.

Suppose $|\lambda-f(e)|<\Sigma_{s \neq e}|f(s)|$. If $\lambda=f(e)$, then clearly $\lambda \in \operatorname{Sp} f$. Hence we may assume that $\lambda \neq f(e)$.

Let $g=1 /[\lambda-f(e)] \cdot(\lambda-f)$. Then $g(e)=1, g$ is free and $\Sigma_{s \neq e}|g(s)|=$ $[1 /|\lambda-f(e)|] \Sigma_{s \neq 1}|f(s)|>1$. Hence, by Lemma 5, $g$ is not invertible in $A$, and thus $\lambda-f$ is not invertible in $A$, i.e. $\lambda \in \operatorname{Sp} f$. Hence $\{\lambda:|\lambda-f(e)|<$ $\left.\Sigma_{s \neq e}|f(s)|\right\} \subset \mathrm{Sp} f$. But since Sp $f$ is closed [2, 1. 6. 4] we get $\{\lambda:|\lambda-f(e)| \leq$ $\left.\Sigma_{s \neq e}|f(s)|\right\} \subset \mathrm{Sp} f$, which completes the proof.

We now give an example of an element of $A$ (necessarily nonfree) that does not satisfy the Theorem.

Let $f(e)=1, f(x)=2 / 3, f\left(x^{2}\right)=4 / 9=(2 / 3)^{2}$ and $f(s)=0$ for all other $s \in F$. Then $f$ has an inverse in $\Gamma$ since $f(e) \neq 0$.

We have $1=f^{-1} f(e)=f^{-1}(e) f(e)=f^{-1}(e)$, and

$$
0=f^{-1} f(x)=f^{-1}(e) f(x)+f^{-1}(x) f(e)=f(x)+f^{-1}(x) .
$$

Hence $f^{-1}(x)=-f(x)=-2 / 3$. For $k>1$, we have

$$
\begin{aligned}
0 & =f^{-1} f\left(x^{k}\right)=f^{-1}\left(x^{k-2}\right) f\left(x^{2}\right)+f^{-1}\left(x^{k-1}\right) f(x)+f^{-1}\left(x^{k}\right) \\
& =(2 / 3)^{2} f^{-1}\left(x^{k-2}\right)+(2 / 3) f^{-1}\left(x^{k-1}\right)+f^{-1}\left(x^{k}\right) .
\end{aligned}
$$

Hence $f^{-1}\left(x^{k}\right)=-(2 / 3)^{2} f^{-1}\left(x^{k-2}\right)-(2 / 3) f^{-1}\left(x^{k-1}\right)$.

Using this iteration formula we get,

$$
\left|f^{-1}\left(x^{k}\right)\right|= \begin{cases}0 & \text { if } k=2(\bmod 3) \\ (2 / 3)^{k} & \text { if } k \neq 2(\bmod 3)\end{cases}
$$

By Lemma $2, f^{-1}(s)=0$ for all $s \neq x^{k}$ for some $k$.

Now $\left\|f^{-1}\right\|<\Sigma_{k=0}^{\infty}(2 / 3)^{k}<\infty$; hence $f^{-1} \in A$, i.e. $0 \notin \operatorname{Sp} f$. Nevertheless, $\Sigma_{s \neq e}|f(s)|=10 / 9>f(e)$.

In closing we mention that all the results above hold (with the same proof) if $F$ was replaced by the free semigroup on $n$ generators for an arbitrary $n$. 


\section{REFERENCES}

1. A. H. Al-Moajil, Nilpotency and quasipotency in Banach algebras, Ph.D. Dissertation, University of Oregon, Corvallis, Ore., 1973.

2. C. E. Rickart, General theory of Banach algebras, University Ser. in Higher Math., Van Nostrand, Princeton, N. J., 1960. MR 22 \#5903.

DEPARTMENT OF MATHEMATICS, UNIVERSITY OF OREGON, EUGENE, OREGON 97403

Current address: Department of Mathematics, University of Petroleum and Minerals, Dhahran, Saudi Arabia 